\title{
Maximum Frictional Dissipation and the Information Entropy of Windspeeds
}

\author{
Ralph D. Lorenz \\ Lunar and Planetary Lab, University of Arizona, Tucson, AZ, USA \\ Communicated by A. Bejan, Durham, NC, USA \\ Registration Number 926
}

\begin{abstract}
A link is developed between the work that thermally-driven winds are capable of performing and the entropy of the windspeed history: this information entropy is minimized when the minimum work required to transport the heat by wind is dissipated. When the system is less constrained, the information entropy of the windspeed record increases as the windspeed fluctuates to dissipate its available work. Fluctuating windspeeds are a means for the system to adjust to a peak in entropy production.
\end{abstract}

\section{Introduction}

It has been long-known that solar heating is the driver of winds [1]. Winds represent the thermodynamic conversion of heat into work: this work is expended in accelerating airmasses, with that kinetic energy ultimately dissipated by turbulent drag. Part of the challenge of the weather forecaster is to predict the speed and direction of wind. In this paper, I explore how the complexity of wind records (and more generally, of velocity descriptions of thermally-driven flow) relates to the available work and the frictional or viscous dissipation.

\section{Zonal Energy Balance}

Two linked heat fluxes drive the Earth's weather - the vertical transport of heat upwards, and the transport of heat from low to high latitudes. In the present paper only horizontal transport is considered, although the concepts may be extended by analogy into the vertical dimension.

The Earth's spherical figure, its obliquity, and the latitudinal distribution of albedo are such that the sunlight absorbed in the tropical half of the Earth's surface is nearly 


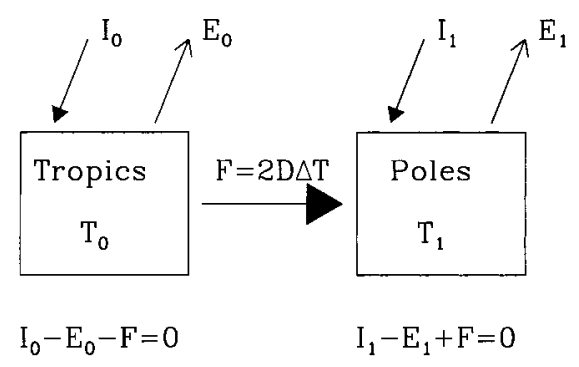

Fig. 1. Schematic of the Earth's climate system, with heat flow $F$ running from low to high latitudes by atmosphere and ocean transport. Energy balance equations for each box can be solved if $I, E(T)$ and $D$ are known. $D$ is in principle determined by the climate system itself via fluid motions.

twice that of the poleward half, on an annual average basis. While higher latitudes are cooler, and hence radiate less to space, the temperature contrast is mitigated by the poleward flow of heat, transported by the oceans and atmosphere. This situation is shown schematically in Figure 1, with $I_{0}, I_{1}$ the absorbed solar fluxes at low and high latitudes, respectively, $F$ the heat flux (heat flow per unit area) of heat from low to high latitudes, and $E=f(T)$ the outgoing thermal emission to space, e.g. some linearization like $E=A+B T$ with $A, B$ constants, or $E=\sigma T^{4}$, with $T$ the temperature and $\sigma$ the Stefan-Boltzmann constant.

In traditional zonal energy balance models (e.g. [2-5]), the latitudinal heat flux $F$ is related to the temperature gradient by a single, empirically-determined, heat transport parameter $D$, which describes the 'effectiveness' of heat transport by the atmosphere ( $D$ is dimensionally equivalent to a heat diffusivity.) In the model expressed in Figure $1, F=2 D \Delta T$. Typically, a value of $D$ of $\sim 0.6 \mathrm{Wm}^{-2} \mathrm{~K}^{-1}$ is used for terrestrial climate studies.

If $D$ is large, heat is 'efficiently' transported from low to high latitudes - were $D$ is infinite, the planet would be nearly isothermal, since energy balance limits the flux $F$ and thus $\Delta T$ becomes inversely related to $D$. On the other hand, if $D$ is very small, the two parts of the planet are in an essentially independent radiative equilibrium and $\Delta T$ tends to a limiting value determined by the radiative constraints $(I, E)$. The work that can be performed by the heat flow in either of these two cases is zero, in the former case because the temperature drop (and therefore the thermodynamic efficiency) is infinitesimal, and in the latter because there is no heat flow to work with. At some value of $D$ in between, however, the available work output has a maximum [6]. Figure 2 shows the low and high latitude temperatures, and available work output, for Earth as a function of $D$.

As can be seen from the shaded boxes in Figure 2 denoting the observed temperatures of the Earth's low and high latitudes, the value of $D$ which yields the observed climate is also the value that maximizes the work available from poleward heat flow. This idea has been pointed out in various forms by a number of workers, notably Paltridge [7] who expressed it as a principle of 'minimum entropy exchange', later as 


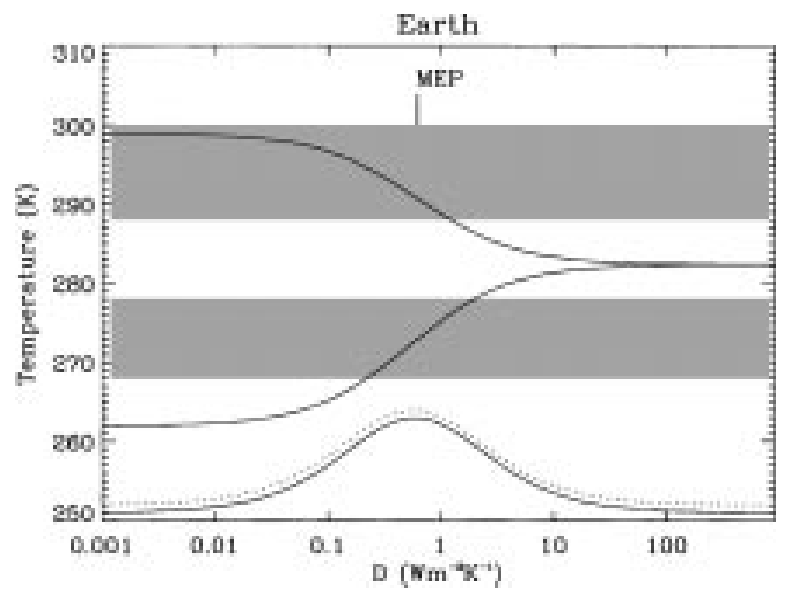

Fig. 2. Terrestrial temperatures at low and high latitudes (solid curves converging to the right) as a function of the transport parameter $D$. Shaded boxes denote observed temperatures. Dotted curve at bottom is entropy production on an arbitrary scale - observed state corresponds to the maximum entropy production ('MEP'). Solid curve that is near-identical to the dotted entropy production is the available work, again on an arbitrary scale.

'maximum entropy production' ('MEP') and Lin [6] as a maximum of available potential energy; Ed Lorenz [8] also explored the idea in an early form. Subsequent models e.g. [9-12] have confirmed that the Earth's climate is indeed in a state that is indistinguishable from this MEP state. It has also been pointed out that this MEP principle also correctly predicts the heat flow and zonal temperatures of other planetary climates, specifically Mars and Titan [13].

It should be noted that the principle is often also called a state of maximum dissipation (which in steady state is the same as maximum work generation [8]) Strictly speaking, the value of $D$ yielding a maximum work production $\left(W=2 D \Delta T^{2} / T_{0}\right)$ is not exactly the same as that yielding a maximum in entropy production $\left(S=F \Delta T / T_{0} T\right)$ : in practice, however, the two cases are nearly indistinguishable (see Fig. 2).

Now, if an idealized atmosphere has a bulk polewards transport velocity $\langle\mathbf{v}\rangle$, then $D=c_{p}\langle\mathbf{v}\rangle P / R g$, where $P / g$ is the pressure divided by gravity, i.e. the column mass of the atmosphere (equivalently $\rho H$, where $\rho$ is the density of the atmosphere at the surface and $H$ is the scale height), $c_{p}$ is the specific heat of air, $R$ is the planetary radius and a characteristic velocity of around $1 \mathrm{~ms}^{-1}$ is obtained [13]. However, the real atmosphere does not flow with a steady $\mathbf{v}$ from hot to cold, and this characteristic velocity $\langle\mathbf{v}\rangle$ only describes the net transport effect, i.e. the average velocity.

Whether the heat transport and thus the average of the atmosphere is determined from an extremum principle or not, the assertion has been made that the atmosphere must somehow dissipate all of the work that the heat flow is capable of producing (e.g. $[14\})$. Brunt $[15,16]$ made an early estimate of the frictional dissipation in the atmosphere of $5 \mathrm{Wm}^{-2}$. It is rarely remarked why this value is so much lower than the 
ideal output of around $12 \mathrm{Wm}^{-2}$, although much of this is due to the vertical rather than the horizontal flow of heat. A likely possibility is that a 'typical' windspeed does not include much of the dissipation, which is concentrated in relatively rare storms not captured in typical runs of large-scale GCMs.

\section{Frictional Dissipation by Variable Winds}

The heat transport relates to the average wind velocity $\langle\mathbf{v}\rangle$ (Speed and direction) while the average cube of windspeed $\left\langle v^{3}\right\rangle$ is what determines frictional dissipation. If both the dissipation and the heat transport are known, then two parameters of the wind speed distribution are known. (Although the ideas may be extended into spatial variations, we consider here only temporal variations of fluid motion at a single location.)

In fact, two-parameter windspeed distributions are widely used in wind energy and hazard studies. One widely-used description is the Weibull distribution, given by the cumulative probability distribution

$$
P(>v)=\exp \left[-(v / c)^{k}\right]
$$

where $c$ is a scale speed, and $k$ is a shape parameter describing the skewness of the distribution and the length of the high-speed tail. When $k=1$, the Weibull distribution reduces to the Rayleigh distribution, and when $k=3.7$ it is equivalent to a Gaussian. For terrestrial hourly-mean windspeeds, scale speeds between 2 and $10 \mathrm{~m} / \mathrm{s}$ and $k$ values of around 2 are typical, tending towards 1.2 for coastal regions. Median wind speed is around $0.75 \mathrm{c}$, whereas the maximum windspeed encountered one-two hundredth of the time (i.e. a 'three sigma' wind) is $\left(5.3^{1 / k}\right) c$ or about $2.5-4 c$

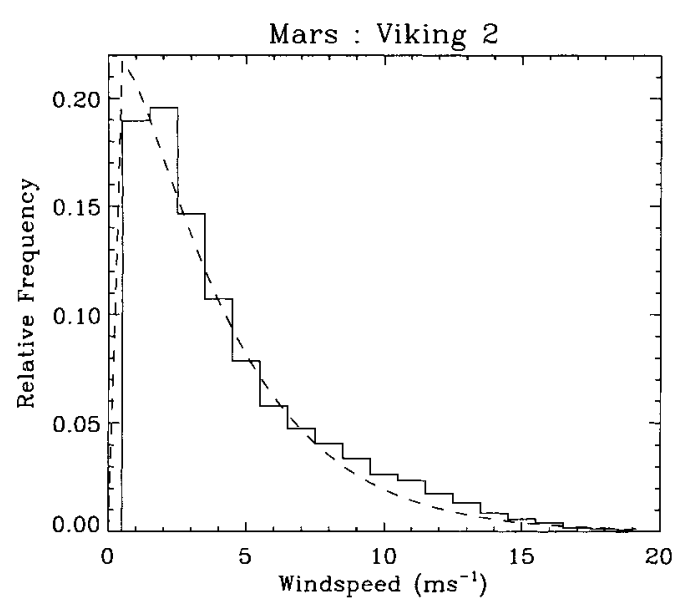

Fig. 3. Martian near-surface windspeeds (solid line) recorded by the Viking 2 lander described by a Weibull distribution (dashed line) with $c=3.8 \mathrm{~m} / \mathrm{s}$ and $k=1.22$. Note the high-speed tail of the distribution. 
for $k=2-1.2$. Near-surface windspeeds on Mars were recorded over a 1050-sol period by the Viking 1 lander; that windspeed record is well-described [18] by Weibull parameters $c=3.85 \mathrm{~m} / \mathrm{s}, k=1.22$ (see Fig. 3.)

The frictional dissipation per unit area $L$ for a given windspeed $v$ is given by $0.5 \rho C_{d} v^{3}$ with $C_{d}$ a drag coefficient, usually $0.002-0.02$. For a given heat flow, all other things being equal, $\langle v\rangle$ must increase as the reciprocal of $\rho$, and thus thinner atmospheres require more dissipation (i.e. work) to transport the heat.

Integrating the Weibull distribution shows that the mean cube-root-mean-cubed speed is higher than the mean speed by a factor of approximately $\left(1+0.8 / k^{3}\right)$. Thus a drop in the value of $k$ from 2.0 to 1.0 can lead to an increase of dissipation - for the same mean windspeed - by more than a factor of 4 . Let us call this factor $\lambda=\left\langle v^{3}\right\rangle /\langle\mathbf{v}\rangle^{3}$. It follows that any form of an averaging (i.e. a loss of information) will reduce the calculated frictional dissipation. Numerical models with coarse grids will therefore underestimate dissipation.

\section{Information Entropy of Windspeed Records}

The foregoing analysis has treated winds as unidirectional - clearly winds may, in fact, fluctuate. Fluctuations that direct winds in opposition to the temperature gradient (i.e. negagive $\mathbf{v}$ ) clearly cause dissipation while requiring the rest of the distribution to be higher to compensate for the negative heat transport. Hence, as for unidirectional winds, an increased available work and thus dissipation for a given heat flow implies a larger variation in wind velocity.

Consider, for example, a site where the wind is always constant, and always in the same direction (from hot to cold). This could equivalently be described by a Weibull distribution with a very large $k$. Since the $\left\langle v^{3}\right\rangle$ is here equal to $\langle\mathbf{v}\rangle^{3}$, this situation develops the minimum possible dissipation for the heat transported. Clearly, for thinner atmospheres or higher drag coefficients, the dissipation would be higher, but we consider these parameters fixed in this discussion.

As another extreme, consider a site where the wind blows at a constant speed, but in both directions with nearly equal probability, say $0.5+\delta$ in the hot-cold direction, and $0.5-\delta$ in the opposite direction. The effective heat transport velocity is therefore $\sim+2 \delta v$. However, the dissipation relates only to the mean cube speed $\left\langle v^{3}\right\rangle$, regardless of direction, here simply $v^{3}$. When $\delta$ is small, it follows that the net heat transport is small, and thus the efficiency (dissipation divided by heat) of the flow must be highest, since the dissipation produced is not dependent on $\delta$.

Each wind measurement in this idealized situation requires one bit of data to specify the $(+$ or -$)$ direction. However, the information content of this bitstream depends on the probability of fluctuations in direction - for the trivial case of $\delta=0.5$ the wind is always in the positive direction, the bitstream is simply a string of ones. Such a bitstream has essentially zero information content (it could be encoded with an arbitrarily high compression factor without loss). The information content can be expressed as the entropy $S$ [19] of the symbol stream $S=-\Sigma p_{i} \log _{2}\left(p_{i}\right)$, where $p_{i}$ is 
the probability of each symbol, $\Sigma p_{i}=1$. Here $S=(0.5-\delta) \log _{2}(0.5-\delta)-(0.5+\delta)$ $\log _{2}(0.5+\delta)$. For $\delta=0.5$, with the lowest dissipation possible for the heat transport, $S=0$. In contrast, if $\delta=0.01$, say, requiring that the thermodynamic efficiency of the flow be 50 times higher, we have a rich information stream, $S=0.9997$. For $\delta=0$, $S=1$ and the wind record is completely incompressible and unpredictable, although this would require an infinite thermodynamic efficiency since work is being done by a zero net flow of heat. The limit on the entropy of the flow, and thus the minimum value of $\delta$, will depend on the physical setting.

Thus, there is a direct relationship between the thermodynamic efficiency of the flow, and the information entropy of the record of the flow. The same relationship will apply to variable windspeeds - distributions with lower values of $k$ span velocity space better (they are more equivalent to a flat symbol frequency distribution) and hence have a higher entropy.

\section{Discussion - Laboratory experiments on Rayleigh-Benard convection}

If the flow pattern and velocity were to remain fixed (e.g. a a single toroidal circulation cell) then the heat flow, described by the Nusselt number $\mathrm{Nu}$, would increase proportionally to the Rayleigh number $R a$. These dimensionless parameters are, respectively, the total heat flow (convective+conductive) divided by the conductive flow, and a measure of the driving buoyancy force divided by the retarding viscosity. The work that the system could perform would increase proportionally to $R a^{2}$, since both the heat flow and the temperature drop (and hence Carnot efficiency) are proportional at $\Delta T$. For fixed flow conditions (and assuming the viscosity does not change), the viscous dissipation would remain constant, and hence at higher $R a$ there would appear an exces work capacity. As is well-known, this does not happen in practice, the circulation pattern, and the boundary layer thickness, adjust such that $\mathrm{Nu}$ is proportional to $R a^{0.3}$.

Although often expressed in terms such as 'the flow adjusts to more efficiently transport heat', what is actually happening is the flow is adjusting to match its dissipation with its available work. In order to do so, the temperature drop across the convecting part drops (lowering the available work), and the flow changes (to increase the dissipation). Specifically, the flow breaks into smaller cells (so introducing more shear per unit area for a given flow speed) and fluctuates in velocity both direction and speed - such that the average velocity drops compared to the average cube speed.

As described in this paper, the mismatch between the minimum dissipation required to transport heat, and the work available form the flow, leads to the generation of information, and hence a more complex flow history. Laboratory data from Niemela et al. [20] show this process in action quantitatively - at low Rayleigh numbers $\left(R a \sim 10^{6}, N u \sim 10\right)$, the velocity distribution measured at a point is unidirectional and strongly peaked, whereas at high $R a\left(\sim 10^{13}, N u \sim 1000\right)$ there is a bidirectional, broad and nearly symmetric velocity distribution. At high $R a$, the flow is more chaotic and unpredictable - the entropy of its probability distribution increases. For 

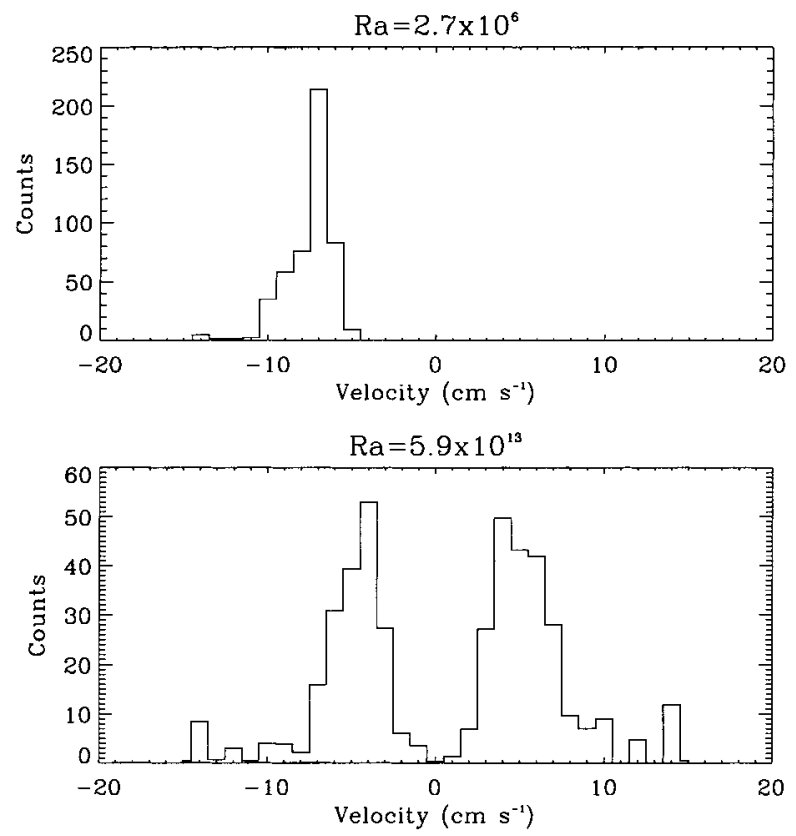

Fig. 4. Flow velocity distributions in covecting helium (Niemela et al., 2000) for low and high Rayleigh numbers. For low $R a$ the flow is unidirectional and fairly narrowly-distributed - an attempt to 'guess' the velocity would likely be correct; at high $R a$ the flow is bidirectional with extended high-speed tails and it is nearly impossible to guess the instantaneous velocity: it also has much higher specific dissipation (higher $\lambda$ ).

these two cases, the mean flow velocities $\langle\mathbf{v}\rangle$ are -7.5 and $+0.80 \mathrm{cms}^{-1}$, respectively. The cube-root-mean-cube speeds $\left\langle v^{3}\right\rangle$ are 7.78 and $6.98 \mathrm{cms}^{-1}$; the high- $R a$ flow dissipates about 8 times more work per net mass transport than the low-Ra flow.

The ideas described here have been pointed out in other forms. For example, numerical simulations of convecting fluids [21] have indicated that the sum of the production of thermodynamic entropy and Kolmogorov entropy is invariant for a given Rayleigh number. Specifically, where two modes of heat transport pertain, one transports more heat, but the other generates more information ('has a higher rate of phase space information loss'). On a more philosophical note, Swenson [22] uses Rayleigh-Benard convection and the successive symmetry-breaking at higher heat flows as a parable for the evolution of complexity in the universe, and associates a law of maximum entropy production to the selection of the flow state.

Fluctuating flow gives the climate system the freedom to select a maximum entropy production state. The system derives kinetic energy from the large-scale flow driven by nonuniform solar heating $[1,8]$. To be in steady-state, the dissipation must grow to match the work being supplied. For an 'efficient' direct circulation, only modest boundary layer drag is available for dissipation. However, fluctuations in windspeed allow dissipation to increase for the same net heat flow - subsequent motions, lower in the energy cascade, accelerate to soak up the available work. This concept is 


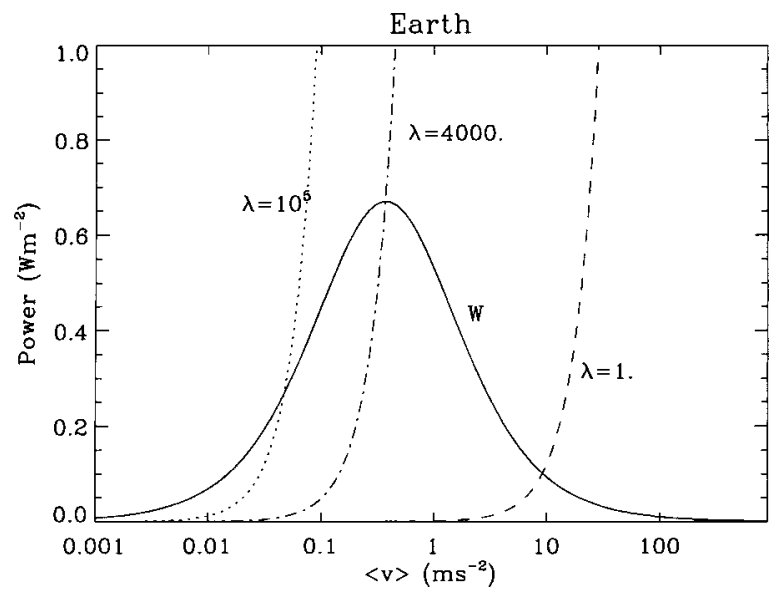

Fig. 5. Work available from latitudinal heat flow on Earth (solid line) as a function of $D$, i.e. $\langle v\rangle$. In steady-state, work output must equal dissipation (other curves) which depends on the speed distribution associated with $\langle v\rangle$, i.e. $\lambda$. For $\lambda=4000$, the steady state occurs at the point of maximum work production.

explored in the work of Malkus [23]. If dissipation increases beyond the present work production, the energy of the flow beings to fall. The temperature difference driving the direct circulation would therefore increase, pushing the system to a state of higher work production via the direct circulation. The net effect of these interactions would be to 'ratchet' the system into a state of maximum dissipation.

The situation is visualized in Figure 5. If only a direct circulation is invoked, $\lambda=1$ and the system would settle at a heat flow beyond that corresponding to maximum work production - where the dissipation vs $\langle v\rangle$ curve intersects the work production curve. This situation is unstable in the sense that the energy would cascade down from this large scale flow to generate eddies and nonuniform velocities, and hence increase $\lambda$. This would continue until $\lambda$ - and the complexity of the flow - had grown to the point where the intersection occurs at the maximum work production. If, because of some physical constraint like a high $C_{d}$, or an arbitrarily thin atmosphere like that of Mercury or Triton requiring high velocity, the dissipation for even the most direct flow $(\lambda=1)$ is very large (shown schematically on Figure 5 as a curve for $\lambda=10^{5}$ ) then the intersection occurs for heat flow lower than that for maximum dissipation or MEP. In these constrained environments the system does not have enough degrees of freedom to reach MEP; on the other hand, these systems are constrained to be simple and have low information entropies, i.e. to be predictable.

\section{Conclusions}

A framework has been set out that relates the complexity or information entropy of a wind speed record to the dissipation produced by those winds, and the thermodynamic entropy produced by the heat transported by them. It is noted that the cubic 
dependence of frictional dissipation on velocity implies that any averaging of velocity over space and time will result in an underestimation of the dissipation.

The production of information relies on the system's being capable of performing more work by transporting heat than is dissipated by viscous friction in so doing. The system selects a more complex flow pattern, with higher dissipation, in order to consume the available work. Bejan [24, 25] has articulated a 'constructional theory' embodying related ideas. Where systems have the freedom afforded by available work, they select a configuration in which their dissipation is maximized - see also $[26,27]$. Systems which are constrained to have heat fluxes below that which leads to maximum dissipation are intrinsically more predictable and produce less information. It follows, for example, that increasing the dissipation (e.g. increasing $C_{d}$, by installing wind turbines) will therefore decrease the work available to be turned into information. Records of fluid flow will never be completely random, since that would imply an unachievable thermodynamic efficiency of unity.

\section{References}

[1] Hadley, G., Concerning the cause of the general tradewinds, Phil. Trans., 36 (1735), $58-62$.

[2] North, G., Cahalan, R.F., Coakley, J.A., Energy balance climate models, Rev. Geophys. Space Phys., 19 (1981), 91-121.

[3] Williams, D., Kasting, J.F., Habitable Planets with high obliquities, Icarus, 129 (1997), 254-267.

[4] Sellers, W.D., A climate model based on the energy balance of the earth-atmosphere system, J. Applied Meteorology, 8 (1969), 392-400.

[5] James, P.B., North, G., The seasonal $\mathrm{CO}_{2}$ cycle on Mars: An application of an energy balance model, Journal of Geophysical Research, 87 (1982), 10271-10283.

[6] Lin, C.A., An extremal principle for a 1-dimensional climate model, Geophys. Res. Lett., 9 (1982), 716-718.

[7] Paltridge, G.W., Global dynamics and climate change: A system of minimum entropy exchange, Q. J. Roy. Met. Soc., 101 (1975), 475-484.

[8] Lorenz, E.N., Generation of available potential energy and the intensity of the general circulation, in: Dynamics of Climate, Ed. R.L. Pfeffer, pp. 86-92, Pergamon Press, New York, 1960.

[9] Grassl, H., The climate at maximum entropy production by meridional atmospheric and ocean heat fluxes, Q. J. Roy. Met. Soc., 107 (1981), 153-1701.

[10] Sohn, B., Smith, E.A., Energy transport by ocean and atmosphere based on an entropy extremum principle. Part 1: Zonal averaged transports, J. Climate, 6 (1993), 886-899.

[11] Shutts, G.J., Maximum entropy production states in quasi-geostrophic dynamical models, Q. J. Roy. Met. Soc., 107 (1981), 503-520.

[12] Pujol, T., Llebot, J.E., Extremal Climatic states simulated by a 2-dimensional model. Part 1: Sensitivity of the model and present state, Tellus, 52A (2000), 422-439.

[13] Lorenz, R.D., Lunine, J.I., McKay, C.P., Withers, P.G., Titan, Mars and Earth: Entropy production by latitudinal heat transport, Geophys. Res. Lett., 25 (2001), 415-418.

[14] Michaud, L.M., Heat to work conversion during upward convection. Part 1: Carnot engine method, Atmospheric Research, 39 (1995), 157-178.

[15] Brunt, D., Physical and Dynamical Meteorology (2 ${ }^{\text {nd }}$ ed.), Cambridge University Press, Cambridge, UK, 1952.

[16] Brunt, D., Energy in the earth's atmosphere, Phil. Mag. S. 7, 1 (1926), 523-532.

[17] Peixoto, J.P., Oort, A.H., The Physics of Climate, American Institute of Physics, New York, 1992. 
[18] Lorenz, R.D., Martian surface wind speeds described by the Weibull distribution, Journal of Spacecraft and Rockets, 33 (1996), 754-756.

[19] Shannon, C., A mathematical theory of communication, Bell System Technical Journal, 27 (1948), 379-423 and 623-656.

[20] Niemela, J.J., Skrbek, L., Sreenivasa, K.R., Donnelly, R.J., Turbulent convection at very high Rayleigh numbers, Nature, 404 (2000), 837-840.

[21] Castillo, V.M., Hoover, W.G., Entropy production and Lyapunov instability at the onset of turbulent convection, Physical Review E., 58 (1998), 7350-7354.

[22] Swenson, R., Emergent attractors and a law of maximum entropy production: Foundations to a theory of general evolution, Systems Research, 6 (1989), 187-197.

[23] Malkus, W.V.R., The heat transport and spectrum of thermal turbulence, Proc. Roy. Soc. A., 225 (1956), 186-195.

[24] Bejan, A., Shape and Structure, from Engineering to Nature, Cambridge University Press, Cambridge, UK, 2000.

[25] Nelson, R.A.Jr., Bejan, A., Constructal optimization of internal flow geometry in convection, ASME Journal of Heat Transfer, 120 (1998), 357-364.

[26] Lorenz, R.D., Planets, life, and the production of entropy, International Journal of Astrobiology, 1 (2002), 3-13.

[27] Ozawa, H., Shimokawa, S., Sakuma, H., Thermodynamics of fluid turbulence: A unified approach to the maximum transport properties, Physical Review E, 64 (2001), 026303-1026303-8.

Recieved 2001-10-09

Accepted 2002-03-15

R.D. Lorenz

Lunar and Planetary Lab,

University of Arizona

1629 E. University Blvd.

Tucson, AZ 85721-0092

USA

E-mail: rlorenz@1pl.arizona.edu 